\title{
Hermite-Hadamard type inequalities for operator convex functions on the co-ordinates
}

\section{ShuHong Wang}

College of Mathematics, Inner Mongolia University for the Nationalities, 028043 Tongliao, China.

Communicated by Sh. Wu

\begin{abstract}
In the paper, the concept of operator convexity on the co-ordinates is introduced and some new Hermite-Hadamard type inequalities for operator convex functions on the co-ordinates are established. (c)2017 All rights reserved.
\end{abstract}

Keywords: Hermite-Hadamard type inequality, co-ordinated operator convex function.

2010 MSC: 15A45, 15A46, 15A47, 47A63, 26B25, 26D15.

\section{Introduction}

Throughout this paper, let $\mathbb{R}=(-\infty, \infty)$ and $\mathbb{R}_{0}=[0, \infty)$.

The following inequality holds for any convex function $f$ defined on $\mathbb{R}$ and $a, b \in \mathbb{R}$ with $a<b$

$$
f\left(\frac{a+b}{2}\right) \leqslant \frac{1}{b-a} \int_{a}^{b} f(x) d x \leqslant \frac{f(a)+f(b)}{2} .
$$

Both inequalities hold in the reversed direction, if $f$ is concave on $[a, b]$. The inequality (1.1) is well-known in the literature as Hermite-Hadamard's inequality. We note that the Hermite-Hadamard's inequality may be regarded as a refinement of the concept of convexity and it follows easily from Jensen's inequality. The classical Hermite-Hadamard's inequality provides estimates of the mean value of a continuous convex function $f:[a, b] \rightarrow \mathbb{R}$.

In [2], Dragomir considered convex functions on the co-ordinates defined in a rectangle from the plane.

Definition $1.1([2])$. Let $\triangle:=[\mathrm{a}, \mathrm{b}] \times[\mathrm{c}, \mathrm{d}]$ be the bidimensional interval in $\mathbb{R}^{2}$ with $\mathrm{a}<\mathrm{b}$ and $\mathrm{c}<\mathrm{d}$. The function $f: \triangle \rightarrow \mathbb{R}$ is said to be convex, if the following inequality

$$
f(\lambda x+(1-\lambda) z, \lambda y+(1-\lambda) w) \leqslant \lambda f(x, y)+(1-\lambda) f(z, w),
$$

holds for all $(x, y),(z, w) \in \triangle$ and $\lambda \in[0,1]$. 
Definition 1.2 ([2]). A function $f: \triangle:=[a, b] \times[c, d] \subseteq \mathbb{R}^{2} \rightarrow \mathbb{R}$ will be called convex on the co-ordinates with $a<b$ and $c<d$, if the partial mappings $f_{y}:[a, b] \rightarrow \mathbb{R}, f_{y}(u):=f(u, y)$ and $f_{x}:[c, d] \rightarrow \mathbb{R}, f_{x}(v):=$ $f(x, v)$ are convex where defined for all $y \in[c, d]$ and $x \in[a, b]$.

A formal definition for co-ordinated convex functions may be stated as follows.

Definition 1.3. Let $\triangle:=[a, b] \times[c, d]$ be the bidimensional interval in $\mathbb{R}^{2}$ with $a<b$ and $c<d$. A function $f: \triangle \rightarrow \mathbb{R}$ will be said to be convex on the co-ordinates on $\triangle$, if the inequality

$$
\begin{aligned}
f(t x+(1-t) z, \lambda y+(1-\lambda) w) & \\
& \leqslant t \lambda f(x, y)+t(1-\lambda) f(x, w)+\lambda(1-t) f(z, y)+(1-t)(1-\lambda) f(z, w),
\end{aligned}
$$

holds for all $(x, y),(x, w),(z, y),(z, w) \in \triangle$ and $t, \lambda \in[0,1]$.

In [2], Dragomir also obtained the following inequalities of Hadamard type.

Theorem 1.4. Suppose that $\mathrm{f}: \triangle=[\mathrm{a}, \mathrm{b}] \times[\mathrm{c}, \mathrm{d}] \subseteq \mathbb{R}^{2} \rightarrow \mathbb{R}$ is convex on the co-ordinates on $\triangle$ with $\mathrm{a}<\mathrm{b}$ and $\mathrm{c}<\mathrm{d}$. Then one has the inequalities

$$
\begin{aligned}
f\left(\frac{a+b}{2}, \frac{c+d}{2}\right) \leqslant & \frac{1}{2}\left[\frac{1}{b-a} \int_{a}^{b} f\left(x, \frac{c+d}{2}\right) d x+\frac{1}{d-c} \int_{c}^{d} f\left(\frac{a+b}{2}, y\right) d y\right] \\
\leqslant & \frac{1}{(b-a)(d-c)} \int_{a}^{b} \int_{c}^{d} f(x, y) d x d y \\
\leqslant & \frac{1}{4}\left[\frac{1}{b-a} \int_{a}^{b} f(x, c) d x+\frac{1}{b-a} \int_{a}^{b} f(x, d) d x\right. \\
& \left.+\frac{1}{d-c} \int_{c}^{d} f(a, y) d y+\frac{1}{d-c} \int_{c}^{d} f(b, y) d y\right] \\
\leqslant & \frac{f(a, c)+f(a, d)+f(b, c)+f(b, d)}{4} .
\end{aligned}
$$

The above inequalities are sharp.

Recently, several extensions and generalizations have been considered for classical convexity. A significant generalization of convex functions is that of operator convex functions introduced by Dragomir in [5].

We review the operator order in $B(H)$ and the continuous functional calculus for a bounded selfadjoint operator. For self-adjoint operators $A, B \in B(H)$, we write $A \leqslant B$, if $\langle A x, x\rangle \leqslant\langle B x, x\rangle$ for every vector $x \in H$, we call it the operator order.

Let $A$ be a bounded self-adjoint linear operator on a complex Hilbert space $(H ;\langle.,\rangle$.$) . The Gelfand map$ establishes a $*$-isometrically isomorphism $\Phi$ between the set $\mathrm{C}(\operatorname{Sp}(A))$ of all continuous complex-valued functions defined on the spectrum of $A$, denoted $S p(A)$ and the $C^{*}$-algebra $C^{*}(A)$ generated by $A$ and the identity operator $1_{H}$ on $H$ as follows (see for instance [20, p.3]). For any $f, g \in C(S p(A)$ ) and any $\alpha, \beta \in \mathbb{C}$, we have

(i) $\Phi(\alpha f+\beta g)=\alpha \Phi(f)+\beta \Phi(g)$;

(ii) $\Phi(f g)=\Phi(f) \Phi(g)$, and $\Phi\left(f^{*}\right)=\Phi(f)^{*}$;

(iii) $\|\Phi(f)\|=\|f\|:=\sup _{t \in \operatorname{Sp}(A)}|f|$;

(iv) $\Phi\left(f_{0}\right)=1_{H}$, and $\Phi\left(f_{1}\right)=A$, where $f_{0}(t)=1$ and $f_{1}(t)=t$ for $t \in S p(A)$.

With this notation, we define

$$
f(A):=\Phi(f), \quad \forall f \in C(S p(A))
$$

and we call it the continuous functional calculus for a bounded self-adjoint operator $A$. 
If $A$ is a bounded self-adjoint operator and $f$ is a real-valued continuous function on $S p(A)$, then $f(t) \geqslant 0$ for any $t \in S p(A)$ implies that $f(A) \geqslant 0$, i.e., $f(A)$ is a positive operator on $H$. Moreover, if both $f$ and $g$ are real-valued functions on $\operatorname{Sp}(A)$ such that $f(t) \leqslant g(t)$ for any $t \in \operatorname{Sp}(A)$, then $f(A) \leqslant f(B)$ in the operator order in $\mathrm{B}(\mathrm{H})$.

A real-valued continuous function $f$ on an interval $I \subseteq \mathbb{R}$ is said to be operator convex (operator concave), if the operator inequality

$$
f((1-\lambda) A+\lambda B) \leqslant(\geqslant)(1-\lambda) f(A)+\lambda f(B),
$$

holds in the operator order in $B(H)$, for all $\lambda \in[0,1]$ and for every bounded self-adjoint operators $A$ and $\mathrm{B}$ in $\mathrm{B}(\mathrm{H})$ whose spectra are contained in $\mathrm{I}$.

In [5], Dragomir gave the operator version of the Hermite-Hadamard inequality for operator convex functions.

Theorem 1.5. Let $\mathrm{f}: \mathrm{I} \subseteq \mathbb{R} \rightarrow \mathbb{R}$ be an operator convex function on the interval $\mathrm{I}$. Then for any self-adjoint operators A and B with spectra in I, we have the inequality

$$
\begin{aligned}
f\left(\frac{A+B}{2}\right) & \leqslant \frac{1}{2}\left[f\left(\frac{3 A+B}{4}\right)+f\left(\frac{A+3 B}{4}\right)\right] \\
& \leqslant \int_{0}^{1} f(t A+(1-t) B) d t \leqslant \frac{1}{2}\left[f\left(\frac{A+B}{2}\right)+\frac{f(A)+f(B)}{2}\right] \leqslant \frac{f(A)+f(B)}{2} .
\end{aligned}
$$

Some inequalities of Hermite-Hadamard type were also obtained in $[1,3,4,6-8,11-14,16-19,21]$ and plenty of references therein.

Motivated by the above results we investigate in this paper operator convex functions on the coordinates and the associated Hermite-Hadamard type inequalities.

\section{Operator convex function on the co-ordinates}

Let $\mathrm{I}_{1}, \mathrm{I}_{2}$ be real intervals and let $\mathrm{f}: \mathrm{I}_{1} \times \mathrm{I}_{2} \rightarrow \mathbb{R}$ be a Borel measurable and essentially bounded function. Let $X=\left(X_{1}, X_{2}\right)$ be a 2-tuple of bounded self-adjoint operators on Hilbert spaces $H_{1}, H_{2}$ such that the spectrum of $X_{i}$ is contained in $I_{i}$ for $i=1,2$. We say that such a 2-tuple is in the domain of $f$. If

$$
X_{i}=\int_{I_{i}} \lambda_{i} E_{i}\left(d \lambda_{i}\right), \quad i=1,2,
$$

is the spectral decomposition of $X_{i}$ where $E_{i}$ is a bounded positive measure on $I_{i}$, we define

$$
f(X)=\int_{I_{1} \times I_{2}} f\left(\lambda_{1}, \lambda_{2}\right) E_{1}\left(d \lambda_{1}\right) \otimes E_{2}\left(d \lambda_{2}\right),
$$

as a bounded self-adjoint operator on the tensor product $\mathrm{H}_{1} \otimes \mathrm{H}_{2}$. If the Hilbert spaces are of finite dimension, then the above integrals become finite sums and we may consider the functional calculus for arbitrary real functions. This construction have the property that

$$
f\left(X_{1}, X_{2}\right)=f_{1}\left(X_{1}\right) \otimes f_{2}\left(X_{2}\right),
$$

whenever $f$ can be separated as a product $f\left(t_{1}, t_{2}\right)=f_{1}\left(t_{1}\right) f_{2}\left(t_{2}\right)$ of two functions each depending on only one variable.

With above functional calculus, we say that a function $f: I_{1} \times I_{2} \rightarrow \mathbb{R}$ is said to be operator convex, if $f$ is continuous and the operator inequality

$$
f(t X+(1-t) Y) \leqslant t f(X)+(1-t) f(Y),
$$


holds for all 2-tuples of self-adjoint operators $X=\left(X_{1}, X_{2}\right)$ and $Y=\left(Y_{1}, Y_{2}\right)$ in the domain of $f$ acting on any Hilbert spaces $\mathrm{H}_{1}, \mathrm{H}_{2}$ and for all $t \in[0,1]$.

For some fundamental results on operator convex and operator monotone functions of several variables, see $[9,10,15]$ and the references therein

Now we define the operator convexity on the co-ordinates.

Definition 2.1. A continuous function $f: I_{1} \times I_{2} \subseteq \mathbb{R}^{2} \rightarrow \mathbb{R}$ is called operator convex on the co-ordinates, if the partial mapping $f_{X_{2}}: I_{1} \rightarrow \mathbb{R}, f_{X_{2}}(u):=f\left(u, X_{2}\right)$ and $f_{X_{1}}: I_{2} \rightarrow \mathbb{R}, f_{X_{1}}(v):=f\left(X_{1}, v\right)$ are operator convex where defined for all operators $X_{2} \in B\left(H_{2}\right)$ and $X_{1} \in B\left(H_{1}\right)$ whose spectra are contained in $I_{2}$ and $\mathrm{I}_{1}$, respectively.

The following is a formal definition of the co-ordinated operator convex function.

Definition 2.2. A continuous function $f: I_{1} \times I_{2} \subseteq \mathbb{R}^{2} \rightarrow \mathbb{R}$ is said to be operator convex on the coordinates, if the operator inequality

$$
\begin{aligned}
f(t A+(1-t) C, & \lambda B+(1-\lambda) D) \\
& \leqslant t \lambda f(A, B)+t(1-\lambda) f(A, D)+(1-t) \lambda f(C, B)+(1-t)(1-\lambda) f(C, D),
\end{aligned}
$$

holds for all $t, \lambda \in[0,1]$ and 2-tuples of self-adjoint operators $(A, B),(C, B),(A, D)$, and $(C, D)$ in the domain of $f$ acting on any Hilbert spaces $\mathrm{H}_{1}, \mathrm{H}_{2}$.

The following lemmas hold:

Lemma 2.3. Every operator convex mapping $\mathrm{f}: \mathrm{I}_{1} \times \mathrm{I}_{2} \subseteq \mathbb{R}^{2} \rightarrow \mathbb{R}$ is operator convex on the co-ordinates, but the converse is not generally true.

Proof. Suppose that $f$ is operator convex mapping on $I_{1} \times I_{2}$. Consider $f_{X_{2}}: I_{1} \rightarrow \mathbb{R}, f_{X_{2}}(u):=f\left(u, X_{2}\right)$. Then for all $t \in[0,1]$ and operators $A, C \in B\left(H_{1}\right)$ with spectra in $I_{1}$, one has

$$
\begin{aligned}
f_{X_{2}}(t A+(1-t) C) & =f\left(t A+(1-t) C, t X_{2}+(1-t) X_{2}\right) \\
& \leqslant t f\left(A, X_{2}\right)+(1-t) f\left(C, X_{2}\right) \\
& =t f_{X_{2}}(A)+(1-t) f_{X_{2}}(C)
\end{aligned}
$$

where $X_{2} \in B\left(H_{2}\right)$ with spectra in $I_{2}$. It shows the operator convexity of $f_{X_{2}}$.

The fact that $f_{X_{1}}: I_{2} \rightarrow \mathbb{R}, f_{X_{1}}(v):=f\left(X_{1}, v\right)$ is also operator convex on $I_{2}$ for all operators $X_{1} \in B\left(H_{1}\right)$ with spectra in $\mathrm{I}_{1}$ goes likewise and we shall omit the details.

Now we consider the function of two variables $f:[0,1]^{2} \rightarrow \mathbb{R}_{0}$ given by $f\left(r_{1}, r_{2}\right)=r_{1} \times r_{2}$. It is obvious that $f$ is operator convex on the co-ordinates but is not operator convex on $[0,1]^{2}$.

Indeed, choosing $A=D=1_{H}, B=C=0_{H}$ and $t \in[0,1]$, we write

$$
\begin{aligned}
f(t A+(1-t) C, t B+(1-t) D) & =(t A+(1-t) C) \otimes(t B+(1-t) D) \\
& =t(1-t) A \otimes D=t(1-t) 1_{H},
\end{aligned}
$$

and

$$
t f(A, B)+(1-t) f(C, D)=t A \otimes B+(1-t) C \otimes D=0_{H}
$$

Thus, for all $t \in(0,1)$, we know

$$
f(t A+(1-t) C, t B+(1-t) D)>t f(A, B)+(1-t) f(C, D),
$$

which shows that $f$ is not operator convex on $[0,1]^{2}$. Lemma 2.3 is thus proved. 
Lemma 2.4. Let $f: I_{1} \times I_{2} \subseteq \mathbb{R}^{2} \rightarrow \mathbb{R}$ be a continuous function on $I_{1} \times I_{2}$. Then for operators $(A, B),(C, D) \in$ $\mathrm{B}\left(\mathrm{H}_{1}\right) \times \mathrm{B}\left(\mathrm{H}_{2}\right)$ with spectra in $\mathrm{I}_{1} \times \mathrm{I}_{2}$, the function $\mathrm{f}$ is operator convex on the co-ordinates for operators in $[A, C]:=\{t A+(1-t) C\} \times[B, D]:=\{\lambda B+(1-\lambda) D\}$ if and only if the function defined by

$$
\varphi_{x, A, B, C, D}(t, \lambda):=\langle f(t A+(1-t) C, \lambda B+(1-\lambda) D) x, x\rangle,
$$

is convex on the co-ordinates on $[0,1] \times[0,1]$ for every $\mathrm{x} \in \mathrm{H}$ with $\|\mathrm{x}\|=1$.

Proof. Suppose that $f$ is operator convex on the co-ordinates for operators in $[A, C] \times[B, D]$, then for any $t_{1}, t_{2}, \lambda_{1}, \lambda_{2} \in[0,1]$ and $\alpha_{i}, \beta_{i} \geqslant 0$ with $\alpha_{i}+\beta_{i}=1$ for $i=1,2$, we drive

$$
\begin{aligned}
\varphi_{x, A, B, C, D}\left(\alpha_{1} t_{1}+\beta_{1} t_{2}, \alpha_{2} \lambda_{1}+\beta_{2} \lambda_{2}\right)= & \left\langlef \left(\alpha_{1}\left(t_{1} A+\left(1-t_{1}\right) C\right)+\beta_{1}\left(t_{2} A+\left(1-t_{2}\right) C\right),\right.\right. \\
& \left.\left.\alpha_{2}\left(\lambda_{1} B+\left(1-\lambda_{1}\right) D\right)+\beta_{2}\left(\lambda_{2} B+\left(1-\lambda_{2}\right) D\right)\right) x, x\right\rangle \\
\leqslant & \alpha_{1} \alpha_{2}\left\langle f\left(t_{1} A+\left(1-t_{1}\right) C, \lambda_{1} B+\left(1-\lambda_{1}\right) D\right) x, x\right\rangle \\
& +\alpha_{1} \beta_{2}\left\langle f\left(t_{1} A+\left(1-t_{1}\right) C, \lambda_{2} B+\left(1-\lambda_{2}\right) D\right) x, x\right\rangle \\
& +\beta_{1} \alpha_{2}\left\langle f\left(t_{2} A+\left(1-t_{2}\right) C, \lambda_{1} B+\left(1-\lambda_{1}\right) D\right) x, x\right\rangle \\
& +\beta_{1} \beta_{2}\left\langle f\left(t_{2} A+\left(1-t_{2}\right) C, \lambda_{2} B+\left(1-\lambda_{2}\right) D\right) x, x\right\rangle \\
= & \alpha_{1} \alpha_{2} \varphi_{x, A, B, C, D}\left(t_{1}, \lambda_{1}\right)+\alpha_{1} \beta_{2} \varphi_{x, A, B, C, D}\left(t_{1}, \lambda_{2}\right) \\
& +\beta_{1} \alpha_{2} \varphi_{x, A, B, C, D}\left(t_{2}, \lambda_{1}\right)+\beta_{1} \beta_{2} \varphi_{x, A, B, C, D}\left(t_{2}, \lambda_{2}\right) .
\end{aligned}
$$

It shows that $\varphi_{x, A, B, C, D}(t, \lambda)$ is convex on the co-ordinates on $[0,1] \times[0,1]$.

Let now $\varphi_{x, A, B, C, D}(t, \lambda)$ be convex on the co-ordinates on $[0,1] \times[0,1]$ and $E=t_{1} A+\left(1-t_{1}\right) C, F=$ $t_{2} A+\left(1-t_{2}\right) C, M=\lambda_{1} B+\left(1-\lambda_{1}\right) D$ and $N=\lambda_{2} B+\left(1-\lambda_{2}\right) D$ for any $t_{1}, t_{2}, \lambda_{1}, \lambda_{2} \in[0,1]$. Fixing $\gamma_{i} \in[0,1], i=1,2$, we find

$$
\begin{aligned}
& \left\langle f\left(\gamma_{1} E+\left(1-\gamma_{1}\right) F, \gamma_{2} M+\left(1-\gamma_{2}\right) N\right) x, x\right\rangle \\
& =\left\langlef \left(\left(\gamma_{1} t_{1}+\left(1-\gamma_{1}\right) t_{2}\right) A+\left(1-\gamma_{1} t_{1}-\left(1-\gamma_{1}\right) t_{2}\right) C,\right.\right. \\
& \left.\left.\left(\gamma_{2} \lambda_{1}+\left(1-\gamma_{2}\right) \lambda_{2}\right) B+\left(1-\gamma_{2} \lambda_{1}-\left(1-\gamma_{2}\right) \lambda_{2}\right) D\right) x, x\right\rangle \\
& =\varphi_{x, A, B, C, D}\left(\gamma_{1} t_{1}+\left(1-\gamma_{1}\right) t_{2}, \gamma_{2} \lambda_{1}+\left(1-\gamma_{2}\right) \lambda_{2}\right) \\
& \leqslant \gamma_{1} \gamma_{2} \varphi_{x, \mathrm{~A}, \mathrm{~B}, \mathrm{C}, \mathrm{D}}\left(\mathrm{t}_{1}, \lambda_{1}\right)+\gamma_{1}\left(1-\gamma_{2}\right) \varphi_{x, \mathrm{~A}, \mathrm{~B}, \mathrm{C}, \mathrm{D}}\left(\mathrm{t}_{1}, \lambda_{2}\right) \\
& +\left(1-\gamma_{1}\right) \gamma_{2} \varphi_{x, A, B, C, D}\left(t_{2}, \lambda_{1}\right)+\left(1-\gamma_{1}\right)\left(1-\gamma_{2}\right) \varphi_{x, A, B, C, D}\left(t_{2}, \lambda_{2}\right) \\
& =\gamma_{1} \gamma_{2}\langle f(E, M) x, x\rangle+\gamma_{1}\left(1-\gamma_{2}\right)\langle f(E, N) x, x\rangle \\
& +\left(1-\gamma_{1}\right) \gamma_{2}\langle f(F, M) x, x\rangle+\left(1-\gamma_{1}\right)\left(1-\gamma_{2}\right)\langle f(F, N) x, x\rangle \text {. }
\end{aligned}
$$

Hence $f$ is operator convex on the co-ordinates and the proof of Lemma 2.4 is complete.

\section{Hermite-Hadamard type inequalities for operator convex functions on the co-ordinates}

In this section, we obtain some new inequalities of Hermite-Hadamard type for the co-ordinated operator convex functions.

Theorem 3.1. Suppose that a continuous function $\mathrm{f}: \mathrm{I}_{1} \times \mathrm{I}_{2} \subseteq \mathbb{R}^{2} \rightarrow \mathbb{R}$ is operator convex on the co-ordinates for all 2-tuples of self-adjoint operators in the domain of $\mathrm{f}$ acting on any Hilbert spaces $\mathrm{H}_{1}, \mathrm{H}_{2}$. Then we have the inequalities

$$
\begin{aligned}
f\left(\frac{A+C}{2}, \frac{B+D}{2}\right) & \leqslant \frac{1}{2}\left[\int_{0}^{1} f\left(t A+(1-t) C, \frac{B+D}{2}\right) d t+\int_{0}^{1} f\left(\frac{A+C}{2}, \lambda B+(1-\lambda) D\right) d \lambda\right] \\
& \leqslant \int_{0}^{1} \int_{0}^{1} f(t A+(1-t) C, \lambda B+(1-\lambda) D) d t d \lambda
\end{aligned}
$$




$$
\begin{aligned}
& \leqslant \frac{1}{4}\left[\int_{0}^{1} f(t A+(1-t) C, B) d t+\int_{0}^{1} f(t A+(1-t) C, D) d t\right. \\
&\left.+\int_{0}^{1} f(A, \lambda B+(1-\lambda) D) d \lambda+\int_{0}^{1} f(C, \lambda B+(1-\lambda) D) d \lambda\right] \\
& \leqslant f(A, B)+f(A, D)+f(C, B)+f(C, D) \\
& 4
\end{aligned}
$$

where $(A, B),(C, D) \in B\left(\mathrm{H}_{1}\right) \times B\left(\mathrm{H}_{2}\right)$ with spectra in $\mathrm{I}_{1} \times \mathrm{I}_{2}$.

Proof. Since the spectrum of $t A+(1-t) C$ and $\lambda B+(1-\lambda) D$ are contained in the intervals $I_{1}$ and $I_{2}$ respectively, and $f$ is continuous, the operator valued integrals $\int_{0}^{1} f(t A+(1-t) C) d t, \int_{0}^{1} f(\lambda B+(1-\lambda) D) d \lambda$ and $\int_{0}^{1} \int_{0}^{1} f(t A+(1-t) C, \lambda B+(1-\lambda) D) d t d \lambda$ exist.

From the co-ordinated operator convexity of $f$ and the inequality (1.2) it is easy to see that

$$
\begin{aligned}
f\left(\frac{A+C}{2}, \lambda B+(1-\lambda) D\right) & \leqslant \int_{0}^{1} f(t A+(1-t) C, \lambda B+(1-\lambda) D) d t \\
& \leqslant \frac{f(A, \lambda B+(1-\lambda) D)+f(C, \lambda B+(1-\lambda) D)}{2} .
\end{aligned}
$$

Integrating this inequality on $[0,1]$ over $\lambda$, we deduce

$$
\begin{aligned}
\int_{0}^{1} f\left(\frac{A+C}{2}, \lambda B+(1-\lambda) D\right) d \lambda & \leqslant \int_{0}^{1} \int_{0}^{1} f(t A+(1-t) C, \lambda B+(1-\lambda) D) d t d \lambda \\
& \leqslant \frac{1}{2}\left[\int_{0}^{1} f(A, \lambda B+(1-\lambda) D) d \lambda+\int_{0}^{1} f(C, \lambda B+(1-\lambda) D) d \lambda\right] .
\end{aligned}
$$

By a similar argument we get

$$
\begin{aligned}
\int_{0}^{1} f\left(t A+(1-t) C, \frac{B+D}{2}\right) d t & \leqslant \int_{0}^{1} \int_{0}^{1} f(t A+(1-t) C, \lambda B+(1-\lambda) D) d t d \lambda \\
& \leqslant \frac{1}{2}\left[\int_{0}^{1} f(t A+(1-t) C, B) d t+\int_{0}^{1} f(t A+(1-t) C, D) d t\right] .
\end{aligned}
$$

Summing the inequalities (3.2) and (3.3) and dividing by 2, we get the second and the third inequalities in (3.1).

Also, by Hermite-Hadamard type inequality (1.2), we observe

$$
\int_{0}^{1} f\left(t A+(1-t) C, \frac{B+D}{2}\right) d t \geqslant f\left(\frac{A+C}{2}, \frac{B+D}{2}\right),
$$

and

$$
\int_{0}^{1} f\left(\frac{A+C}{2}, \lambda B+(1-\lambda) D\right) d \lambda \geqslant f\left(\frac{A+C}{2}, \frac{B+D}{2}\right),
$$

which give, by addition, the first inequality in (3.1).

Finally, by the same inequality we can also state

$$
\begin{aligned}
& \int_{0}^{1} f(t A+(1-t) C, B) d t \leqslant \frac{f(A, B)+f(C, B)}{2} \\
& \int_{0}^{1} f(t A+(1-t) C, D) d t \leqslant \frac{f(A, D)+f(C, D)}{2}
\end{aligned}
$$




$$
\int_{0}^{1} f(A, \lambda B+(1-\lambda) D) d \lambda \leqslant \frac{f(A, B)+f(A, D)}{2}
$$

and

$$
\int_{0}^{1} f(C, \lambda B+(1-\lambda) D) d \lambda \leqslant \frac{f(C, B)+f(C, D)}{2}
$$

which give, by addition, the last inequality in (3.1). The proof thus is complete.

Theorem 3.2. Suppose that continuous functions $\mathrm{f}, \mathrm{g}: \mathrm{I}_{1} \times \mathrm{I}_{2} \subseteq \mathbb{R}^{2} \rightarrow \mathbb{R}$ are operator convex on the co-ordinates for all 2-tuples of self-adjoint operators in the domain of $\mathrm{f}, \mathrm{g}$ acting on any Hilbert spaces $\mathrm{H}_{1}, \mathrm{H}_{2}$. Then for any self-adjoint operators $(\mathrm{A}, \mathrm{B}),(\mathrm{C}, \mathrm{D}) \in \mathrm{B}\left(\mathrm{H}_{1}\right) \times \mathrm{B}\left(\mathrm{H}_{2}\right)$ with spectra in $\mathrm{I}_{1} \times \mathrm{I}_{2}$, we have the inequalities

$$
\begin{gathered}
\int_{0}^{1} \int_{0}^{1}\langle f(t A+(1-t) C, \lambda B+(1-\lambda) D) x, x\rangle\langle g(t A+(1-t) C, \lambda B+(1-\lambda) D) x, x\rangle d t d \lambda \\
\leqslant \frac{4 U(A, C, B, D)+V(A, C, B, D)+2 P(A, C, B, D)}{36}
\end{gathered}
$$

holds for any $\mathrm{x} \in \mathrm{H}$ with $\|\mathrm{x}\|=1$, where

$$
\begin{aligned}
& \mathrm{U}(\mathrm{A}, \mathrm{C}, \mathrm{B}, \mathrm{D})=\langle\mathrm{f}(\mathrm{A}, \mathrm{B}) \mathrm{x}, \mathrm{x}\rangle\langle\mathrm{g}(\mathrm{A}, \mathrm{B}) \mathrm{x}, \mathrm{x}\rangle+\langle\mathrm{f}(\mathrm{A}, \mathrm{D}) \mathrm{x}, \mathrm{x}\rangle\langle\mathrm{g}(\mathrm{A}, \mathrm{D}) \mathrm{x}, \mathrm{x}\rangle \\
& +\langle f(C, B) x, x\rangle\langle g(C, B) x, x\rangle+\langle f(C, D) x, x\rangle\langle g(C, D) x, x\rangle, \\
& \mathrm{V}(\mathrm{A}, \mathrm{C}, \mathrm{B}, \mathrm{D})=\langle\mathrm{f}(\mathrm{A}, \mathrm{B}) \mathrm{x}, \mathrm{x}\rangle\langle\mathrm{g}(\mathrm{C}, \mathrm{D}) \mathrm{x}, \mathrm{x}\rangle+\langle\mathrm{f}(\mathrm{A}, \mathrm{D}) \mathrm{x}, \mathrm{x}\rangle\langle\mathrm{g}(\mathrm{C}, \mathrm{B}) \mathrm{x}, \mathrm{x}\rangle \\
& +\langle f(C, B) x, x\rangle\langle g(A, D) x, x\rangle+\langle f(C, D) x, x\rangle\langle g(A, B) x, x\rangle, \\
& P(A, C, B, D)=\langle f(A, B) x, x\rangle\langle g(A, D) x, x\rangle+\langle f(A, B) x, x\rangle\langle g(C, B) x, x\rangle \\
& +\langle f(A, D) x, x\rangle\langle g(A, B) x, x\rangle+\langle f(A, D) x, x\rangle\langle g(C, D) x, x\rangle \\
& +\langle f(C, B) x, x\rangle\langle g(A, B) x, x\rangle+\langle f(C, B) x, x\rangle\langle g(C, D) x, x\rangle \\
& +\langle\mathrm{f}(\mathrm{C}, \mathrm{D}) \mathrm{x}, \mathrm{x}\rangle\langle\mathrm{g}(\mathrm{A}, \mathrm{D}) \mathrm{x}, \mathrm{x}\rangle+\langle\mathrm{f}(\mathrm{C}, \mathrm{D}) \mathrm{x}, \mathrm{x}\rangle\langle\mathrm{g}(\mathrm{C}, \mathrm{B}) \mathrm{x}, \mathrm{x}\rangle .
\end{aligned}
$$

Proof. Since $f, g$ are operator convex on the co-ordinates, for every $t, \lambda \in[0,1]$ we drive

$$
\begin{aligned}
& \langle f(t A+(1-t) C, \lambda B+(1-\lambda) D) x, x\rangle\langle g(t A+(1-t) C, \lambda B+(1-\lambda) D) x, x\rangle \\
& \leqslant t^{2} \lambda^{2}\langle f(A, B) x, x\rangle\langle g(A, B) x, x\rangle+t^{2} \lambda(1-\lambda)\langle f(A, B) x, x\rangle\langle g(A, D) x, x\rangle \\
& +\mathrm{t}(1-\mathrm{t}) \lambda^{2}\langle\mathrm{f}(\mathrm{A}, \mathrm{B}) \mathrm{x}, \mathrm{x}\rangle\langle\mathrm{g}(\mathrm{C}, \mathrm{B}) \mathrm{x}, \mathrm{x}\rangle+\mathrm{t} \lambda(1-\mathrm{t})(1-\lambda)\langle\mathrm{f}(\mathrm{A}, \mathrm{B}) \mathrm{x}, \mathrm{x}\rangle\langle\mathrm{g}(\mathrm{C}, \mathrm{D}) \mathrm{x}, \mathrm{x}\rangle \\
& +t^{2} \lambda(1-\lambda)\langle f(A, D) x, x\rangle\langle g(A, B) x, x\rangle+t^{2}(1-\lambda)^{2}\langle f(A, D) x, x\rangle\langle g(A, D) x, x\rangle \\
& +t(1-t) \lambda(1-\lambda)\langle f(A, D) x, x\rangle\langle g(C, B) x, x\rangle+t(1-t)(1-\lambda)^{2}\langle f(A, D) x, x\rangle\langle g(C, D) x, x\rangle \\
& +t(1-t) \lambda^{2}\langle f(C, B) x, x\rangle\langle g(A, B) x, x\rangle+t(1-t) \lambda(1-\lambda)\langle f(C, B) x, x\rangle\langle g(A, D) x, x\rangle \\
& +(1-t)^{2} \lambda^{2}\langle f(C, B) x, x\rangle\langle g(C, B) x, x\rangle+(1-t)^{2} \lambda(1-\lambda)\langle f(C, B) x, x\rangle\langle g(C, D) x, x\rangle \\
& +t(1-t) \lambda(1-\lambda)\langle f(C, D) x, x\rangle\langle g(A, B) x, x\rangle+t(1-t)(1-\lambda)^{2}\langle f(C, D) x, x\rangle\langle g(A, D) x, x\rangle \\
& +(1-t)^{2} \lambda(1-\lambda)\langle f(C, D) x, x\rangle\langle g(C, B) x, x\rangle+(1-t)^{2}(1-\lambda)^{2}\langle f(C, D) x, x\rangle\langle g(C, D) x, x\rangle .
\end{aligned}
$$

Integrating both sides of (3.8) over $t, \lambda \in[0,1]$, we get the required inequality (3.4), which completes the proof of Theorem 3.2.

Theorem 3.3. Suppose that continuous functions $\mathrm{f}, \mathrm{g}: \mathrm{I}_{1} \times \mathrm{I}_{2} \subseteq \mathbb{R}^{2} \rightarrow \mathbb{R}$ are operator convex on the co-ordinates for all 2-tuples of self-adjoint operators in the domain of $\mathrm{f}, \mathrm{g}$ acting on any Hilbert spaces $\mathrm{H}_{1}, \mathrm{H}_{2}$. Then for any 
self-adjoint operators $(\mathrm{A}, \mathrm{B}),(\mathrm{C}, \mathrm{D}) \in \mathrm{B}\left(\mathrm{H}_{1}\right) \times \mathrm{B}\left(\mathrm{H}_{2}\right)$ with spectra in $\mathrm{I}_{1} \times \mathrm{I}_{2}$, we have the inequalities

$$
\begin{aligned}
\left\langlef \left(\frac{A+C}{2}\right.\right. & \left.\left., \frac{B+D}{2}\right) x, x\right\rangle\left\langle g\left(\frac{A+C}{2}, \frac{B+D}{2}\right) x, x\right\rangle \\
\leqslant & \frac{1}{4} \int_{0}^{1} \int_{0}^{1}\langle f(t A+(1-t) C, \lambda B+(1-\lambda) D) x, x\rangle\langle g(t A+(1-t) C, \lambda B+(1-\lambda) D) x, x\rangle d t d \lambda \\
& +\frac{5 \mathrm{U}(A, C, B, D)+8 V(A, C, B, D)+7 P(A, C, B, D)}{144},
\end{aligned}
$$

holds for any $\mathrm{x} \in \mathrm{H}$ with $\|\mathrm{x}\|=1$, where $\mathrm{U}(\mathrm{A}, \mathrm{C}, \mathrm{B}, \mathrm{D}), \mathrm{V}(\mathrm{A}, \mathrm{C}, \mathrm{B}, \mathrm{D})$ and $\mathrm{P}(\mathrm{A}, \mathrm{C}, \mathrm{B}, \mathrm{D})$ are defined in (3.5), (3.6) and (3.7), respectively.

Proof. Using the co-ordinated operator convexity of $f, g$, for every $t, \lambda \in[0,1]$ we can write

$$
\begin{aligned}
& \left\langle f\left(\frac{A+C}{2}, \frac{B+D}{2}\right) x, x\right\rangle\left\langle g\left(\frac{A+C}{2}, \frac{B+D}{2}\right) x, x\right\rangle \\
& \leqslant \frac{1}{4}[\langle f(t A+(1-t) C, \lambda B+(1-\lambda) D) x, x\rangle+\langle f(t A+(1-t) C,(1-\lambda) B+\lambda D) x, x\rangle \\
& +\langle f((1-t) A+t C, \lambda B+(1-\lambda) D) x, x\rangle+\langle f((1-t) A+t C,(1-\lambda) B+\lambda D) x, x\rangle] \\
& \times \frac{1}{4}[\langle g(t A+(1-t) C, \lambda B+(1-\lambda) D) x, x\rangle+\langle g(t A+(1-t) C,(1-\lambda) B+\lambda D) x, x\rangle \\
& +\langle g((1-t) A+t C, \lambda B+(1-\lambda) D) x, x\rangle+\langle g((1-t) A+t C,(1-\lambda) B+\lambda D) x, x\rangle] \\
& \leqslant \frac{1}{16}[\langle f(t A+(1-t) C, \lambda B+(1-\lambda) D) x, x\rangle\langle g(t A+(1-t) C, \lambda B+(1-\lambda) D) x, x\rangle \\
& +\langle f(t A+(1-t) C,(1-\lambda) B+\lambda D) x, x\rangle\langle g(t A+(1-t) C,(1-\lambda) B+\lambda D) x, x\rangle \\
& +\langle\mathrm{f}((1-\mathrm{t}) A+\mathrm{tC}, \lambda \mathrm{B}+(1-\lambda) \mathrm{D}) \mathrm{x}, \mathrm{x}\rangle \mathrm{g}((1-\mathrm{t}) \mathrm{A}+\mathrm{tC}, \lambda \mathrm{B}+(1-\lambda) \mathrm{D}) \mathrm{x}, \mathrm{x}\rangle \\
& +\langle\mathrm{f}((1-\mathrm{t}) \mathrm{A}+\mathrm{tC},(1-\lambda) \mathrm{B}+\lambda \mathrm{D}) \mathrm{x}, \mathrm{x}\rangle\langle\mathrm{g}((1-\mathrm{t}) \mathrm{A}+\mathrm{tC},(1-\lambda) \mathrm{B}+\lambda \mathrm{D}) \mathrm{x}, \mathrm{x}\rangle] \\
& +\frac{1}{4}\left\{\mathrm{t}^{2} \lambda^{2}[\langle f(A, B) x, x\rangle\langle g(A, D) x, x\rangle+\langle f(A, B) x, x\rangle\langle g(C, D) x, x\rangle\right. \\
& +\langle f(A, B) x, x\rangle\langle g(C, B) x, x\rangle]+t^{2}(1-\lambda)^{2}[\langle f(A, D) x, x\rangle\langle g(A, B) x, x\rangle \\
& +\langle f(A, D) x, x\rangle\langle g(C, D) x, x\rangle+\langle f(A, D) x, x\rangle\langle g(C, B) x, x\rangle] \\
& +(1-t)^{2} \lambda^{2}[\langle f(C, B) x, x\rangle\langle g(C, D) x, x\rangle+\langle f(C, B) x, x\rangle\langle g(A, B) x, x\rangle \\
& +\langle f(C, B) x, x\rangle\langle g(A, D) x, x\rangle]+(1-t)^{2}(1-\lambda)^{2}[\langle f(C, D) x, x\rangle\langle g(C, B) x, x\rangle \\
& +\langle f(C, D) x, x\rangle\langle g(A, D) x, x\rangle+\langle f(C, D) x, x\rangle\langle g(A, B) x, x\rangle] \\
& +\mathfrak{t}(1-\mathrm{t}) \lambda^{2}[\langle f(A, B) x, x\rangle\langle g(A, D) x, x\rangle+\langle f(A, B) x, x\rangle\langle g(C, D) x, x\rangle \\
& +\langle f(A, B) x, x\rangle\langle g(A, B) x, x\rangle+\langle f(C, B) x, x\rangle\langle g(C, D) x, x\rangle+\langle f(C, B) x, x\rangle\langle g(A, D) x, x\rangle \\
& +\langle f(C, B) x, x\rangle\langle g(C, B) x, x\rangle]+t^{2} \lambda(1-\lambda)[\langle f(A, B) x, x\rangle\langle g(C, B) x, x\rangle \\
& +\langle f(A, B) x, x\rangle\langle g(C, D) x, x\rangle+\langle f(A, B) x, x\rangle\langle g(A, B) x, x\rangle+\langle f(A, D) x, x\rangle\langle g(C, D) x, x\rangle \\
& +\langle f(A, D) x, x\rangle\langle g(A, D) x, x\rangle+\langle f(A, D) x, x\rangle\langle g(C, B) x, x\rangle] \\
& +\mathfrak{t}(1-t)(1-\lambda)^{2}[\langle f(A, D) x, x\rangle\langle g(C, B) x, x\rangle+\langle f(A, D) x, x\rangle\langle g(A, D) x, x\rangle \\
& +\langle f(A, D) x, x\rangle\langle g(A, B) x, x\rangle+\langle f(C, D) x, x\rangle\langle g(C, D) x, x\rangle+\langle f(C, D) x, x\rangle\langle g(A, B) x, x\rangle \\
& +\langle f(C, D) x, x\rangle\langle g(C, B) x, x\rangle]+(1-t)^{2} \lambda(1-\lambda)[\langle f(C, B) x, x\rangle\langle g(A, D) x, x\rangle \\
& +\langle\mathrm{f}(\mathrm{C}, \mathrm{B}) \mathrm{x}, \mathrm{x}\rangle\langle\mathrm{g}(\mathrm{C}, \mathrm{B}) \mathrm{x}, \mathrm{x}\rangle+\langle\mathrm{f}(\mathrm{C}, \mathrm{B}) \mathrm{x}, \mathrm{x}\rangle\langle\mathrm{g}(\mathrm{A}, \mathrm{B}) \mathrm{x}, \mathrm{x}\rangle+\langle\mathrm{f}(\mathrm{C}, \mathrm{D}) \mathrm{x}, \mathrm{x}\rangle\langle\mathrm{g}(\mathrm{C}, \mathrm{D}) \mathrm{x}, \mathrm{x}\rangle \\
& +\langle f(C, D) x, x\rangle\langle g(A, B) x, x\rangle+\langle f(C, D) x, x\rangle\langle g(A, D) x, x\rangle]+t(1-t) \lambda(1-\lambda)(P+U)\} .
\end{aligned}
$$

We integrate both sides of (3.10) over $t, \lambda \in[0,1]$ and obtain the required inequality (3.9), which completes the proof of Theorem 3.3. 
Theorem 3.4. Suppose that continuous functions $\mathrm{f}, \mathrm{g}: \mathrm{I}_{1} \times \mathrm{I}_{2} \subseteq \mathbb{R}^{2} \rightarrow \mathbb{R}$ are operator convex on the co-ordinates for all 2-tuples of self-adjoint operators in the domain of $\mathrm{f}, \mathrm{g}$ acting on any Hilbert spaces $\mathrm{H}_{1}, \mathrm{H}_{2}$. Then for any self-adjoint operators $(\mathrm{A}, \mathrm{B}),(\mathrm{C}, \mathrm{D}) \in \mathrm{B}\left(\mathrm{H}_{1}\right) \times \mathrm{B}\left(\mathrm{H}_{2}\right)$ with spectra in $\mathrm{I}_{1} \times \mathrm{I}_{2}$, we have the inequalities

$$
\begin{aligned}
\left\langlef \left(\frac{A+C}{2},\right.\right. & \left.\left.\frac{B+D}{2}\right) x, x\right\rangle \int_{0}^{1} \int_{0}^{1}\langle g(t A+(1-t) C, \lambda B+(1-\lambda) D) x, x\rangle d t d \lambda \\
& +\left\langle g\left(\frac{A+C}{2}, \frac{B+D}{2}\right) x, x\right\rangle \int_{0}^{1} \int_{0}^{1}\langle f(t A+(1-t) C, \lambda B+(1-\lambda) D) x, x\rangle d t d \lambda \\
\leqslant & \frac{1}{2} \int_{0}^{1} \int_{0}^{1}\langle f(t A+(1-t) C, \lambda B+(1-\lambda) D) x, x\rangle\langle g(t A+(1-t) C, \lambda B+(1-\lambda) D) x, x\rangle d t d \lambda \\
& +\frac{5 U(A, C, B, D)+8 V(A, C, B, D)+7 P(A, C, B, D)}{72},
\end{aligned}
$$

holds for any $\mathrm{x} \in \mathrm{H}$ with $\|\mathrm{x}\|=1$, where $\mathrm{U}(\mathrm{A}, \mathrm{C}, \mathrm{B}, \mathrm{D}), \mathrm{V}(\mathrm{A}, \mathrm{C}, \mathrm{B}, \mathrm{D})$ and $\mathrm{P}(\mathrm{A}, \mathrm{C}, \mathrm{B}, \mathrm{D})$ are defined in (3.5), (3.6) and (3.7), respectively.

Proof. By the co-ordinated operator convexity of $f, g$, for every $t, \lambda \in[0,1]$ we observe that

$$
\begin{aligned}
\left\langlef \left(\frac{A+C}{2},\right.\right. & \left.\left.\frac{B+D}{2}\right) x, x\right\rangle \\
\leqslant & \frac{1}{4}[\langle f(t A+(1-t) C, \lambda B+(1-\lambda) D) x, x\rangle+\langle f(t A+(1-t) C,(1-\lambda) B+\lambda D) x, x\rangle \\
& +\langle f((1-t) A+t C, \lambda B+(1-\lambda) D) x, x\rangle+\langle f((1-t) A+t C,(1-\lambda) B+\lambda D) x, x\rangle]
\end{aligned}
$$

and

$$
\begin{aligned}
\left\langleg \left(\frac{A+C}{2},\right.\right. & \left.\left.\frac{B+D}{2}\right) x, x\right\rangle \\
\leqslant & \frac{1}{4}[\langle g(t A+(1-t) C, \lambda B+(1-\lambda) D) x, x\rangle+\langle g(t A+(1-t) C,(1-\lambda) B+\lambda D) x, x\rangle \\
& +\langle g((1-t) A+t C, \lambda B+(1-\lambda) D) x, x\rangle+\langle g((1-t) A+t C,(1-\lambda) B+\lambda D) x, x\rangle] .
\end{aligned}
$$

We multiply by one under the other and by one across the other of the above inequality and then we add these inequalities, so we obtain

$$
\begin{aligned}
\frac{1}{4}\left\langlef \left(\frac{A+C}{2},\right.\right. & \left.\left.\frac{B+D}{2}\right) x, x\right\rangle[\langle g(t A+(1-t) C, \lambda B+(1-\lambda) D) x, x\rangle \\
& +\langle g(t A+(1-t) C,(1-\lambda) B+\lambda D) x, x\rangle+\langle g((1-t) A+t C, \lambda B+(1-\lambda) D) x, x\rangle \\
& +\langle g((1-t) A+t C,(1-\lambda) B+\lambda D) x, x\rangle]+\frac{1}{4}\left\langle g\left(\frac{A+C}{2}, \frac{B+D}{2}\right) x, x\right\rangle \\
& \times[\langle f(t A+(1-t) C, \lambda B+(1-\lambda) D) x, x\rangle+\langle f(t A+(1-t) C,(1-\lambda) B+\lambda D) x, x\rangle \\
& +\langle f((1-t) A+t C, \lambda B+(1-\lambda) D) x, x\rangle+\langle f((1-t) A+t C,(1-\lambda) B+\lambda D) x, x\rangle] \\
\leqslant & \frac{1}{8}[\langle f(t A+(1-t) C, \lambda B+(1-\lambda) D) x, x\rangle+\langle f(t A+(1-t) C,(1-\lambda) B+\lambda D) x, x\rangle \\
& +\langle f((1-t) A+t C, \lambda B+(1-\lambda) D) x, x\rangle+\langle f((1-t) A+t C,(1-\lambda) B+\lambda D) x, x\rangle] \\
& \times[\langle g(t A+(1-t) C, \lambda B+(1-\lambda) D) x, x\rangle+\langle g(t A+(1-t) C,(1-\lambda) B+\lambda D) x, x\rangle \\
& +\langle g((1-t) A+t C, \lambda B+(1-\lambda) D) x, x\rangle+\langle g((1-t) A+t C,(1-\lambda) B+\lambda D) x, x\rangle] .
\end{aligned}
$$

From the same calculation as in (3.10), we may get (3.11). The details are omitted. 


\section{Acknowledgment}

This work was supported by the Foundation of the Research Program of Science and Technology at Universities of Inner Mongolia Autonomous Region (No. NJZZ16175) and by the Science Research Funding of Inner Mongolia University for Nationalities (No. NMDGP1714 and No. NMDYB1748). The author is thankful to the referee for giving valuable comments and suggestions which helped to improve the final version of this paper.

\section{References}

[1] M. Alomari, M. Darus, The Hadamard's inequality for s-convex function of 2-variables on the co-ordinates, Int. J. Math. Anal. (Ruse), 2 (2008), 629-638. 1

[2] S. S. Dragomir, On the Hadamard's inequality for convex functions on the co-ordinates in a rectangle from the plane, Taiwanese J. Math., 5 (2001), 775-788. 1, 1.1, 1.2, 1

[3] S. S. Dragomir, An inequality improving the first Hermite-Hadamard inequality for convex functions defined on linear spaces and applications for semi-inner products, JIPAM. J. Inequal. Pure Appl. Math., 3 (2002), 8 pages. 1

[4] S. S. Dragomir, An inequality improving the second Hermite-Hadamard inequality for convex functions defined on linear spaces and applications for semi-inner products, JIPAM. J. Inequal. Pure Appl. Math., 3 (2002), 8 pages. 1

[5] S. S. Dragomir, Hermite-Hadamard's type inequalities for operator convex functions, Applied Mathematics and Computation, 218 (2011), 766-772. 1

[6] S. S. Dragomir, S. Fitzpatrick, The Hadamard inequalities for s-convex functions in the second sense, Demonstratio Math., 32 (1999), 687-696. 1

[7] A. G. Ghazanfari, Some new Hermite-Hadamard type inequalities for two operator convex functions, ArXiv, 2012 (2012), 12 pages.

[8] A. G. Ghazanfari, The Hermite-Hadamard type inequalities for operator s-convex functions, J. Adv. Res. Pure Math., 6 (2014), 52-61. 1

[9] F. Hansen, Operator convex functions of several variables, Publ. Res. Inst. Math. Sci., 33 (1997), 443-463. 2

[10] F. Hansen, Operator monotone functions of several variables, Math. Inequal. Appl., 6 (2003), 1-17. 2

[11] K.-C. Hsu, Some Hermite-Hadamard type inequalities for differentiable co-ordinated convex functions and applications, Adv. Pure Math., 4 (2014), 326-340. 1

[12] K.-C. Hsu, Refinements of Hermite-Hadamard type inequalities for differentiable co-ordinated convex functions and applications, Taiwanese J. Math., 19 (2015), 133-157.

[13] H. Hudzik, L. Maligranda, Some remarks on s-convex functions, Aequationes Math., 48 (1994), $100-111$.

[14] E. Kikianty, Hermite-Hadamard inequality in the geometry of Banach spaces, PhD thesis, Victoria University, (2010). 1

[15] A. Korányi, On some classes of analytic functions of several variables, Trans. Amer. Math. Soc., 101 (1961), 520-554. 2

[16] M. A. Latif, M. Alomari, Hadamard-type inequalities for product two convex functions on the co-ordinates, Int. Math. Forum, 4 (2009), 2327-2338. 1

[17] M. A. Latif, M. Alomari, On Hadmard-type inequalities for h-convex functions on the co-ordinates, Int. J. Math. Anal. (Ruse), 3 (2009), 1645-1656.

[18] M. A. Latif, S. S. Dragomir, On some new inequalities for differentiable co-ordinated convex functions, J. Inequal. Appl., 2012 (2012), 13 pages.

[19] S.-L. Lyu, On the Hermite-Hadamard inequality for convex functions of two variables, Numer. Algebra Control Optim., 4 (2014), 1-8. 1

[20] J. Pečarić, T. Furuta, J. Mićić Hot, Y. Seo, Mond-Pečarić method in operator inequalities, Inequalities for bounded selfadjoint operators on a Hilbert space, Monographs in Inequalities, ELEMENT, Zagreb, (2005). 1

[21] M. Z. Sarıkaya, E. Set, M. E. Ozdemir, S. S. Dragomir, New some Hadamard's type inequalities for co-ordinated convex functions, Tamsui Oxf. J. Inf. Math. Sci., 28 (2012), 137-152. 1 